\title{
Long-term follow-up of patients with choroidal neovascularization due to angioid streaks
}

\author{
This article was published in the following Dove Press journal: \\ Clinical Ophthalmology \\ 19 December 2016 \\ Number of times this article has been viewed
}

Maria Guadalupe Martinez-

Serrano'

Abelardo Rodriguez-Reyes ${ }^{2}$

Jose Luis Guerrero-

Naranjo ${ }^{1,3}$

Guillermo Salcedo-

Villanueva'

Jans Fromow-Guerra ${ }^{1,3}$

Gerardo García-Aguirre ${ }^{1,3}$

Virgilio Morales-Canton'

Raul Velez-Montoya ${ }^{1,3}$

'Retina Department, ${ }^{2}$ Pathology

Department, Asociación para Evitar la

Ceguera en Mexico, Hospital "Dr Luis

Sanchez Bulnes" IAP, ${ }^{3}$ Macula Retina

Consultants, Mexico City, Mexico
Correspondence: Raul Velez-Montoya Retina Department, Asociación para

Evitar la Ceguera en Mexico, Hospital

"Dr Luis Sanchez Bulnes" IAP, Calle

Vicente García Torres 46, San Lucas

Coyoacán, 04030,

México City, México

Tel +52 $55 \quad 084 \quad 1400$

$\mathrm{Fax}+5255 \quad 1084 \quad 1404$

Email rvelezmx@yahoo.com
Background: The following case series describes the long-term anatomical and functional outcome of a group of seven patients with choroidal neovascularization (CNV), secondary to angioid streaks (AS), who were treated with antiangiogenic drugs in a pro re nata (PRN) regimen. After the 4-year mark, visual acuity tends to return to pretreatment level. Treatment delays and lack of awareness and self-referral by the patients are believed to be the cause of the PRN regimen failure.

Purpose: To assess the long-term outcomes ( $>4$ years) of patients with CNV due to AS treated with a PRN regimen of antiangiogenic.

Methods: This was a retrospective, case series, single-center study. We reviewed the electronic medical records from patients with CNV due to AS. From each record, we noted general demographic data and relevant medical history; clinical presentation, changes in bestcorrected visual acuity (BCVA) over time, optical coherent tomography parameters, treatment and retreatment details, and systemic associations. Changes in BCVA and central macular thickness were assessed with a Wilcoxon two-sample test, with an alpha value of $\leq 0.05$ for statistical significance.

Results: The mean follow-up time was $53.8 \pm 26.8$ months. BCVA at baseline was: $1.001 \pm 0.62$ $\log$ MAR; at the end of follow-up: $0.996 \pm 0.56 \log$ MAR $(P=0.9)$. Central macular thickness at baseline was: $360.85 \pm 173.82 \mu \mathrm{m}$; at the end of follow-up: $323.85 \pm 100.34 \mu \mathrm{m}(P=0.6)$. Mean number of intravitreal angiogenic drugs: $6 \pm 4.16$ injections (range 4-15). Mean time between injections was 3.8 \pm 2.7 months (range 1.9-5.8 months).

Conclusion: Despite initial anatomical and functional improvement, patients at the end of the follow-up had no visual improvement after a pro re nata regimen of antiangiogenic drugs. The amount of retreatments, number of recurrences, and time between intravitreal injections were similar to previous reports with shorter follow-up.

Keywords: angioid streaks, choroidal neovascularization, long term, antiangiogenic, bevacizumab, ranibizumab, outcome

\section{Introduction}

Angioid streaks (AS) are linear breaks of a calcified Bruch membrane, typically of reddish to brownish-gray color and ragged borders of variable width. ${ }^{1-3}$ They are often bilateral and located in the posterior pole. ${ }^{1,4}$ They typically seem to surround and radiate from the optic nerve head toward retinal periphery. ${ }^{1,2,5}$ This particular localization is believed to be the result of the mechanical stress exerted over a brittle and less flexible posterior pole by the extraocular muscles. ${ }^{1,2}$

The etiology is still not well understood; however, the common occurrence of phenotypically similar AS among various systemic diseases, such as Paget's disease, Ehlers-Danlos syndrome, and various blood dyscrasias (sickle cell disease and beta-thalassemia), ${ }^{1,5-7}$ suggests a common molecular mechanism encompassing 
antimineralization pathways. ${ }^{1,8}$ Possible candidates include mutations in $A B C C 6$ gene transporter protein (multidrug resistance-associated protein 6), which is generally accepted to induce Pseudoxanthoma elasticum (PXE), the systemic disease with the highest association with AS, with an incidence ranging from $59 \%$ to $87 \%$ depending on whether the diagnosis is made clinically or with skin biopsy. $1,4,8$

Even though AS have no initial repercussion on visual function by itself and mostly appear to be stationary, 2,9 $42 \%-86 \%$ of patients with AS will develop choroidal neovascularization (AS-CNV) during the natural history of the disease. ${ }^{5,10}$ This may lead to severe and fast visual deterioration in middle-aged working patients, due to exudation, hemorrhage, and subsequent subretinal fibrosis and atrophy., ${ }^{7,11}$ If left untreated, visual prognosis is poor, often leading to legal blindness and huge economic repercussions due to visual disabilities on a relatively young population. ${ }^{5,6,12}$

Treatment options have included laser photocoagulation, photodynamic therapy, and intravitreal anti-vascular endothelial growth factor drugs. ${ }^{5,9,11,13}$ The latter are the only ones that have proven to be able to induce visual stabilization or increase visual acuity up to 3 years after onset of the treatment. ${ }^{11,14}$ Nevertheless, there is no definitive information regarding which antiangiogenic treatment regimen is the best option, since there are not enough information about the treatment success rate in the long term. Furthermore, results can also be confounded by a high rate of recurrence of AS-CNV, atrophic changes, and progression of fibrosis. ${ }^{1}$

In the current study, we will review the clinical course of a group of patients with AS who developed AS-CNV and were treated with anti-vascular endothelial growth factor drugs in a pro re nata (PRN) scheme, with a minimum follow-up of at least 4 years. The aim of this study is to assess the long-term efficacy of the PRN protocol in terms of visual acuity stabilization as well as to describe AS progression and atrophy formation in the study of eye and AS-CNV incidence in the contralateral eye.

\section{Materials and methods}

This was a retrospective, single-center case series study. This study was approved by the local internal review board (Comité de Investigación, Asociación para Evitar la Ceguera en México, Mexico Ministry of Health). This study was conducted according to the tenets of the Declaration of Helsinki and Good Clinical Practice guidelines. All sensitive data were managed according to the Health Insurance Portability and Accountability Act rules of 1996 and its Mexican equivalent, the Federal Law for Protection of Personal Data in Possession of Individuals (NOM-024-SSA3-2010).
Due to the retrospective nature of the study, no written informed consent from the participants was required by the review board.

We reviewed all electronic medical records in the retina department from 2005 to 2011 . The study included only those records with a clinical diagnosis of AS and/or AS-CNV in at least one eye (study eye), and those which were treated with a PRN regimen of intravitreal anti-vascular endothelial growth factor drugs. There were no restrictions regarding refractive error or concomitant eye diseases unless it would have interfered with the visual acuity assessment or required surgery during the surveyed time frame. This study excluded all medical records of patients who did not meet a minimum of at least 4 years of reported office visits, disregarding missed appointments; medical records from patients with CNV due to other causes, different to AS; and medical records from patients who underwent a treat and extend regime.

From each included medical record, we extracted the following information: general demographic data, relevant medical history, time between symptoms onset and clinical diagnosis, history of previous treatments, current treatments including the type of antiangiogenic drug, dose, number of retreatments, and time between injections. In addition, from each ophthalmological examination registered, we reviewed all data concerning the following: best-corrected visual acuity (BCVA) - converted into logarithm of the minimum angle of resolution equivalents (logMAR) for statistical analysis. Visual acuities of count fingers equaled to $1.7 \log$ MAR, hand movement to $2.0 \log$ MAR, light perception to $2.3 \log M A R$, and no light perception to $3.0 \operatorname{logMAR} .{ }^{15}$ From ancillary tests we noted the angiographic pattern and the anatomical localization of leakage of dye, in relation to the fovea and AS, by fluorescein angiography (FA). Central macular thickness (CMT) and CNV anatomical classification were established by optical coherent tomography (OCT). Data from admission until their last follow-up examination on record were reviewed for both eyes.

Statistical analysis was done using an excel spreadsheet (Excel 2007; Microsoft Corp., Redmond, WA, USA). Changes in BCVA and central macular thickness were assessed with a Wilcoxon two-sample test, with an alpha value of $\leq 0.05$ for statistical significance.

\section{Results}

We reviewed the data of 14 eyes with AS, belonging to 7 patients ( 3 males, 4 females) who fulfilled all the inclusion/exclusion criteria on at least one of their eyes. Six right eyes and one left eye were labeled as study eyes because they had AS-CNV as the cause of their present illness during 
admission to the retina department. During the follow-up, 3 contralateral eyes developed AS-CNV and were treated with antiangiogenic drugs as well. The information regarding the contralateral eyes was analyzed separately. All cases were treatment naïve.

The mean age at presentation was $53.2 \pm 8.4$ years. Three patients reported acute decrease of vision as the main complaint during admission; two reported central scotoma as the main complaint; and two reported persistent metamorphopsia as the main reason for coming into the hospital. Patients with metamorphopsia as chief complaint were the first to seek help within 1 month from the onset of symptom. They were also slightly younger than the other 5 patients (47 and 52 years of age). Patients who reported decrease of vision as chief complaint looked for help within the first 12 months from the onset of symptoms. The ages were 44, 53, and 56 years. Finally, patients with central scotoma as chief complaint were the last to seek help, waiting up to 21 months before coming into the hospital. The ages were 71 and 50 years. The mean follow-up time was 53.8 \pm 26.8 months (range 50-120 months). The average follow-up time equated to $\sim 4.4$ years (Figure 1).

The most prevalent anatomical location of AS-CNV in the study eye was subfoveal (5 out of 7), of which, 1 patient referred metamorphopsia as the chief complaint, 2 more mentioned central scotoma as the chief complaint, and the last 2 referred vision loss as the principal complaint. The other 2 study eyes had juxtafoveal localization of the AS-CNV and complained of metamorphopsia and decreased vision, respectively. Furthermore, in 5 out of 7, the CNV were in close proximity (in contact) with the path of the AS; and seemed to develop from the AS. The other two AS-CNVs were close to an AS, but not directly in contact with them. The angiographic patterns and OCT localization, as well as the presence of retinal pigment epithelium atrophy or subretinal fibrosis at presentation, are summarized in Table 1. At the end of the follow-up, all patients had significant subretinal fibrosis.

During baseline examination, three patients had concomitant peau d'orange fundus appearance and "comet-trail" atrophic spots in the midperiphery. All 3 were confirmed to have PXE by skin biopsy ( $43 \%$ of the patients). There were no other systemic diseases associated with AS on this series (Figure 2).

The mean BCVA in the study eyes at baseline was $1.001 \pm 0.62 \log$ MAR $(\approx 20 / 200)$. The mean BCVA at the end of the follow-up was $0.996 \pm 0.56 \log \mathrm{MAR}(\approx 20 / 198)$. There were no differences between baseline and last mean $\operatorname{BCVA}(P=0.9)$. CMT in the study eyes was $360.85 \pm 173.82 \mu \mathrm{m}$ at baseline and $323.85 \pm 100.34 \mu \mathrm{m}$ at the end of follow-up.

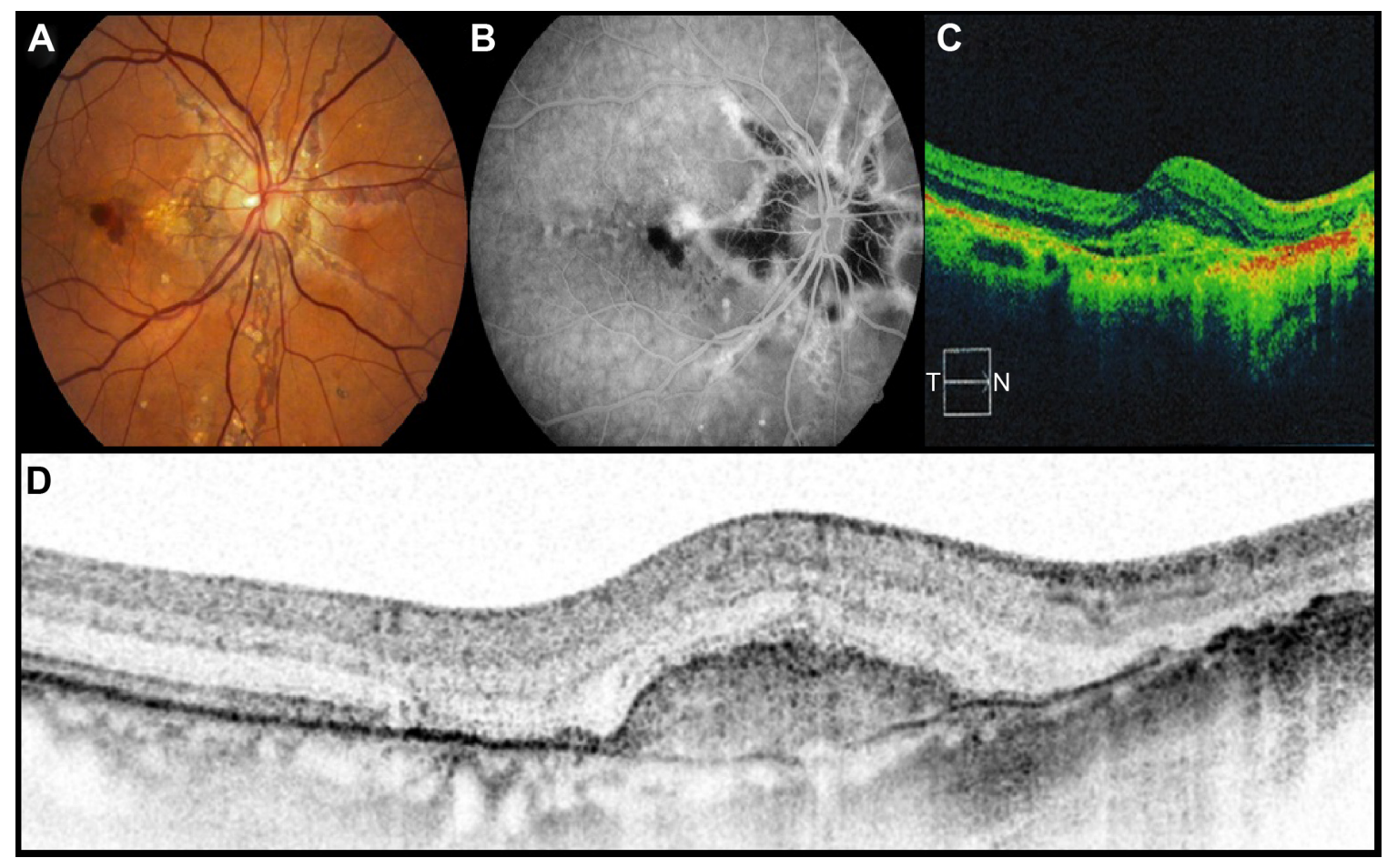

Figure I Case 3. Forty-seven-year-old female who complained of metamorphopsia and decrease of visual acuity as chief complaint.

Notes: (A) Color fundus photograph which shows subretinal hemorrhage associated to angioid streak. (B) Fluorescein angiography, late frames; the figure shows leakage of the dye and blockage due to subretinal hemorrhage. (C) Baseline OCT shows a Type I CNV with subretinal fluid. (D) Macular OCT at the end of the follow-up after 7 doses of intravitreal bevacizumab, which shows a juxtafoveal inactive type I AS-CNV. The final visual acuity was 20/40.

Abbreviations: AS, angioid streaks; CNV, choroidal neovascularization; N, nasal; OCT, optical coherent tomography; T, temporal. 
Table I Angiographic and OCT patterns

\begin{tabular}{|c|c|c|c|c|c|c|c|c|}
\hline \multicolumn{5}{|l|}{ Baseline } & \multicolumn{4}{|c|}{ Last follow-up } \\
\hline $\begin{array}{l}\text { Patient ID } \\
\text { number }\end{array}$ & Chief complaint & Localization & Angiography & OCT type & $\begin{array}{l}\text { RPE } \\
\text { atrophy }\end{array}$ & $\begin{array}{l}\text { Subretinal } \\
\text { fibrosis }\end{array}$ & $\begin{array}{l}\text { RPE } \\
\text { atrophy }\end{array}$ & $\begin{array}{l}\text { Subretinal } \\
\text { fibrosis }\end{array}$ \\
\hline I & Metamorphopsia & Juxtafoveal & Classic & I & - & - & + & + \\
\hline 2 & Vision loss & Juxtafoveal & Predominantly classic & I & - & + & - & + \\
\hline 3 & Metamorphopsia & Subfoveal & Occult & I & - & + & - & + \\
\hline 4 & Scotoma & Subfoveal & Minimally classic & 2 & - & + & - & + \\
\hline 5 & Scotoma & Subfoveal & Predominantly classic & I & + & + & + & + \\
\hline 6 & Vision loss & Subfoveal & Classic & 2 & - & - & - & + \\
\hline 7 & Vision loss & Subfoveal & Predominantly classic & I & - & + & + & + \\
\hline
\end{tabular}

Notes: Summary of the anatomical localization in relation to the fovea of the AS-CNV, chief complaint, angiography patterns, and localization by OCT. At baseline, 5 out of 7 patients had some degree of subretinal fibrosis and only one showed retinal pigment epithelium atrophy. At the end of the follow-up, all 7 eyes had some degree of subretinal fibrosis, from which three had significant retinal pigment epithelium atrophy.

Abbreviations: -, absent; +, present; AS-CNV, angioid streaks-choroidal neovascularization; OCT, optical coherent tomography; RPE, retinal pigment epithelium.

There were no differences between baseline and last CMT $(P=0.6)$.

All patients received one loading dose of either bevacizumab $(1.25 \mathrm{mg} / 0.05 \mathrm{~mL})$ or ranibizumab $(0.5 \mathrm{mg} / 0.05 \mathrm{~mL})$, followed by a PRN scheme. Drug selection was done according to physician preferences. The patients were followed monthly until AS-CNV inactivation characterized by absence of exudation/hemorrhage by FA and OCT and visual stabilization. Retreatments were done according to the visual assessments in every follow-up visit (loss of 5 or more letters from the last visit) or signs of AS-CNV activity by OCT (increased macular thickness or development of new intraretinal or subretinal fluid/hemorrhages regardless of its extension). All patients were instructed to seek immediate help in case of visual symptoms, metamorphopsia, or vision loss.

Six out of 7 study eyes experienced a significant improvement in BCVA (15 or more letters on Snellen charts) after the first intravitreal injections, of which, 50\% required at least three intravitreal doses of antiangiogenic drug before starting to improve. The other 50\% improved BCVA (or decreased symptoms) after a single intravitreal injection.

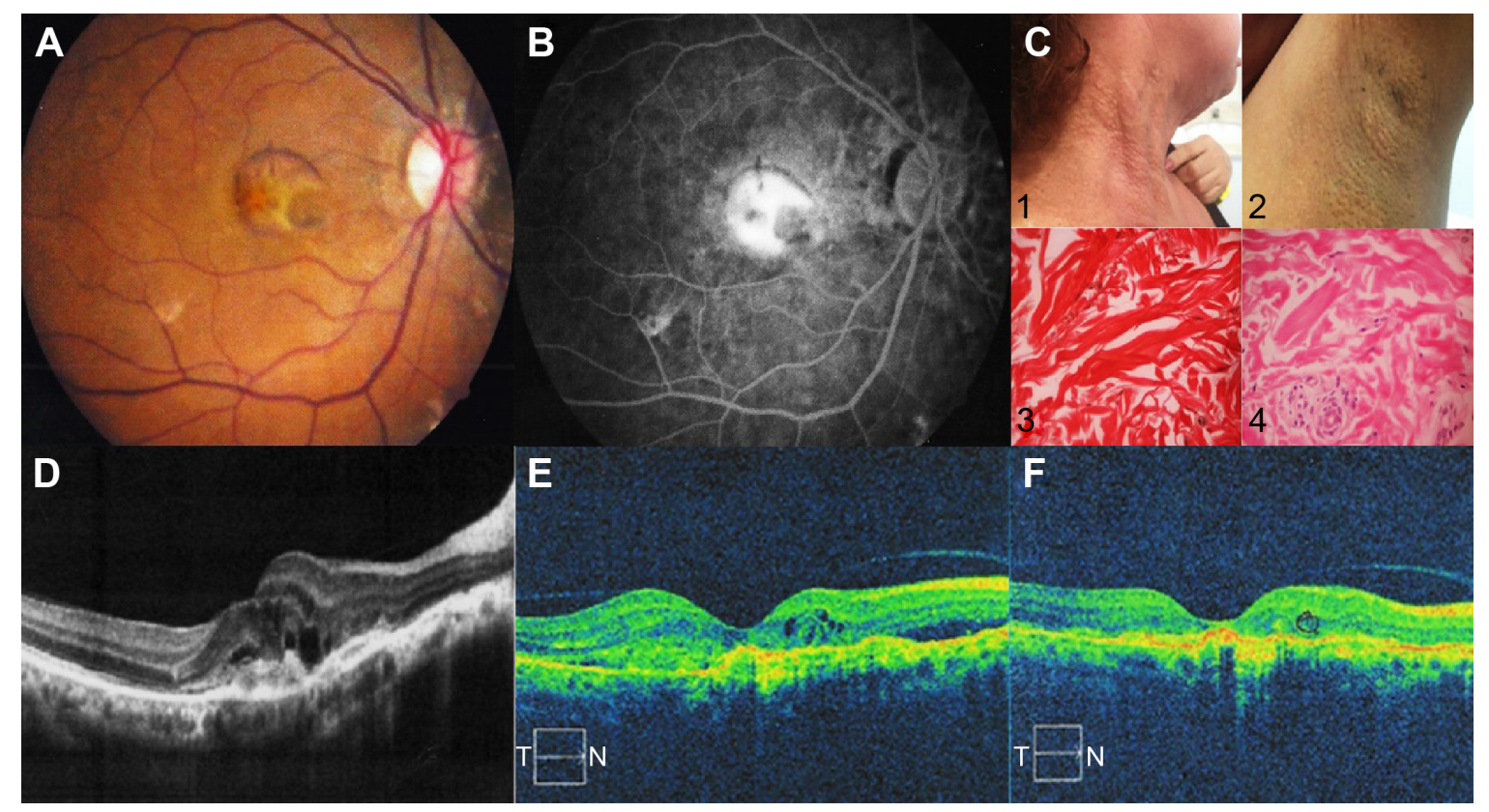

Figure 2 Case 6. Fifty-three-year-old female who complained of decreased visual acuity as chief complaint.

Notes: The BCVA at baseline was 20/250. Images A and B show a subfoveal classic AS-CNV and peau d'orange. CI and C2 show nodular skin lesions (cobblestone appearance) in the lateral part of the neck and axillae. C3, 10× microphotograph, Verhoeff-Van Gieson stain for elastic fibers. Degenerative changes in elastic fibers. C4, IOX microphotograph (hematoxylin and eosin). Irregular elastic fibers (size and shape) and a sweet gland. (D) Baseline OCT showing what seems to be a type 2 CNV with intraretinal fluid. During the follow-up and after repeated OCT studies (E), the type was reclassified as type I. (F) Last follow-up OCT after 7 doses of intravitreal bevacizumab and 2 doses of intravitreal ranibizumab (4 reactivations of the AS-CNV) and 4.5 years of follow-up. The final BCVA was 20/800.

Abbreviations: AS-CNV, angioid streaks-choroidal neovascularization; BCVA, best-corrected visual acuity; N, nasal; OCT, optical coherent tomography; T, temporal. 
Only 1 out of 7 study eyes got worse after the first intravitreal injection and required 5 intravitreal injections before starting to improve BCVA. However, it never regained the same baseline BCVA.

Through the follow-up time, the range of BCVA among the 6 eyes that improved initially was very wide (20/32 to count fingers 4 feet) and vary from visit to visit, depending on if the AS-CNV was inactive or active. All 7 eyes in the study group experienced recurrence of the original AS-CNV at some point of the follow-up (mean: 3.1 1 1.2 times). Although every time the AS-CNV responded to one or two additional doses of intravitreal antiangiogenic, the eye struggled to regain prior BCVA and ended up losing few letters per episode. At the end of the follow-up, all eyes in the study group have some level of subretinal fibrosis (Figure 3 ).

The overall number of AS-CNV recurrence (new loss of 5 or more letters from the last visit or new intraretinal or subretinal fluid/hemorrhages) during the first year of treatment was $0.8 \pm 0.3$; during the second year of treatment was $1.1 \pm 0.6$; during the third year of treatment was $1.0 \pm 0.7$; during the fourth year of treatment was $0.6 \pm 0.5$; and during the fifth year of treatment was $0.6 \pm 0.8$.

All study eyes received intravitreal bevacizumab as the initial antiangiogenic drug. Three of the eyes were switched to ranibizumab during the follow-up in order to improve therapy effectiveness (BCVA). The mean number of bevacizumab injection on the study eyes was $5.4 \pm 3.2$ injections (range 2-9). The mean number of ranibizumab in the 3 study eyes was $1.3 \pm 0.5$ injections (in addition to bevacizumab). Taking into account all antiangiogenic drugs used, regardless if it was bevacizumab or ranibizumab, the overall number of antiangiogenic intravitreal injections were $6 \pm 4.16$ (range 4-15). The mean time between intravitreal injections was very variable among eyes in the study group and was 3.8 \pm 2.7 months (range 1.9-5.8 months).

During the first year of treatment, the overall number of intravitreal injections was 2.2 \pm 0.9 ; during the second year of treatment was $2.0 \pm 1.4$; during the third year of treatment was $2.3 \pm 0.5$; during the fourth year of treatment was $1.0 \pm 1.4$; and during the fifth year of treatment was 0.6 \pm 1.5 .

Regarding the seven contralateral eyes, the mean BCVA at baseline was $0.3903 \pm 0.42(\approx 20 / 45$, range $20 / 20-20 / 150)$. At the end of the follow-up the mean BCVA was $0.4552 \pm 0.44$ $(\approx 20 / 57$, range $20 / 30$ to hand movement). There were no differences between baseline and last mean $\operatorname{BCVA}(P=0.3)$. Three eyes developed AS-CNV during the follow-up (43\%), of which the baseline BCVA was 20/20 (logMAR: 1), 20/25 (logMAR 0.0969), and 20/150 (0.176). At the end

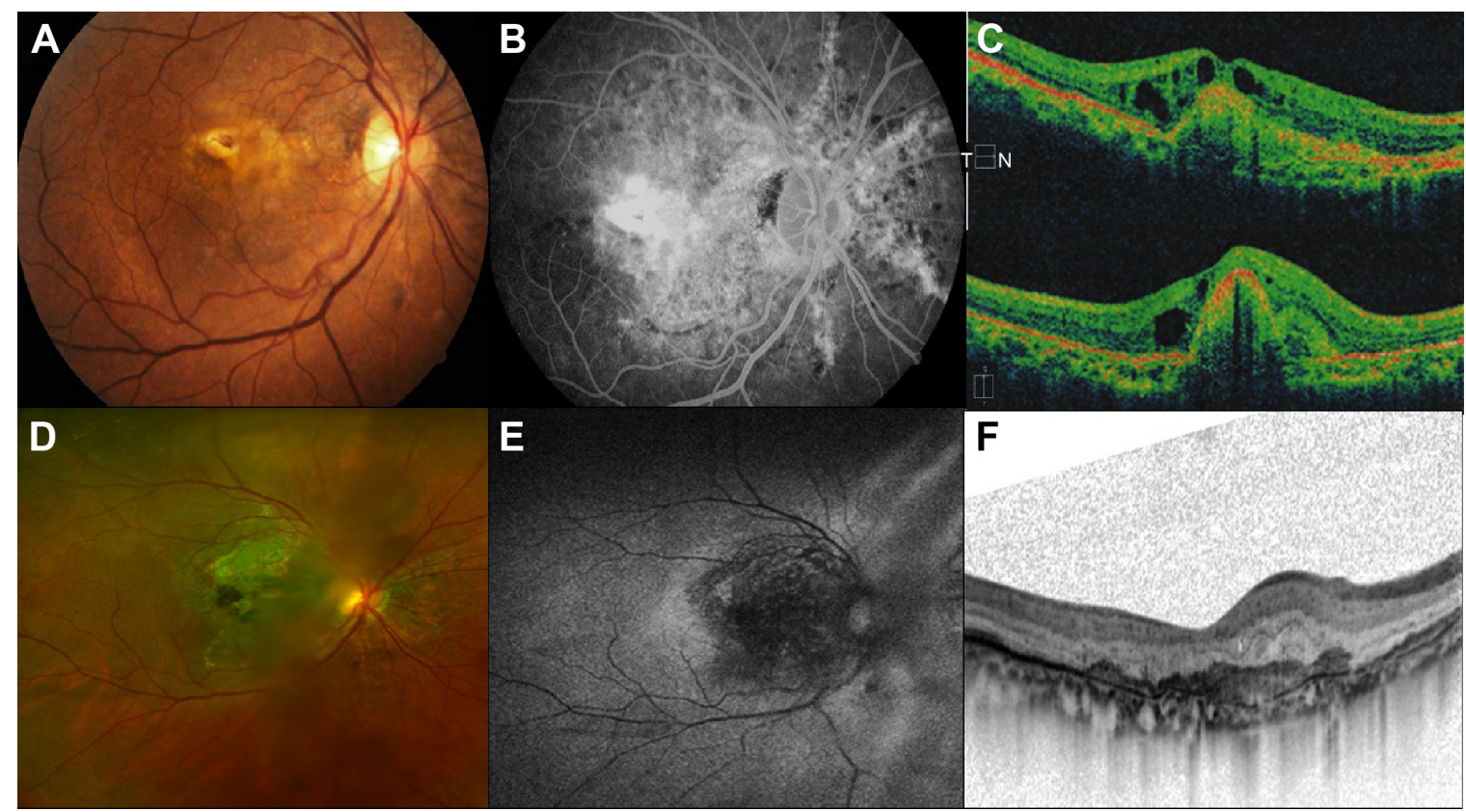

Figure 3 Case I. Fifty-two-year-old male who complained of metamorphopsia and decrease of visual acuity as chief complaint.

Notes: BCVA at baseline: count fingers at 4 feet. (A) Color fundus photograph shows subfoveal AS-CNV. (B) Fluorescein angiography that shows a classic hyperfluorescence pattern. (C) Baseline OCT showing type I CNV and intraretinal fluid. (D) Wide-field color fundus photograph, (E) autofluorescence, and (F) OCT taken at the last follow-up visit. The fundus photographs show important retinal pigment epithelium atrophy and subretinal fibrosis. The autofluorescence shows an increase in the macular hypoautofluorescence. The OCT shows inactive AS-CNV. Final BCVA was 20/I25 after 6 years of follow-up ( 5 relapses of the AS-CNV), 8 intravitreal doses of bevacizumab, and I of ranibizumab.

Abbreviations: AS-CNV, angioid streaks-choroidal neovascularization; BCVA, best-corrected visual acuity; N, nasal; OCT, optical coherent tomography; T, temporal. 
of the follow-up, the BCVA on those three eyes was hand movement (logMAR: 1.3), 20/40 (logMAR: 0.301), and 20/80 (logMAR: 0.602). All three had subfoveal localization, Type 2 AS-CNV on the OCT and classic appearance on FA. The contralateral eyes developed AS-CNV 3.7, 10, and 51.6 months (4.3 years) after the initial diagnosis of AS-CNV on the study eye. The CMT in the three contralateral eyes, which developed AS-CNV at baseline, were 176, 218, and $236 \mu \mathrm{m}$. At the end of the follow-up, the CMT was 220, 356, and $386 \mu \mathrm{m}$. All three received intravitreal bevacizumab as the antiangiogenic of choice ( 2 doses, 4 doses, and 2 doses). One of them developed severe subretinal fibrosis at the end of the follow-up. All AS-CNV were inactive by FA and OCT at the last follow-up visit.

\section{Discussion}

The development of CNV is a major cause of severe visual loss in patient with AS, who are usually young and economically active people. ${ }^{16,17}$ The high level of CNV recurrences through the natural history of the disease represent a real challenge to the treatment. ${ }^{1}$ Even after years of inactivity, new CNV can develop either from a preexisting lesion or in different location, away from the primary lesion. ${ }^{11}$ If left untreated, it can progress to legal blindness due to retina scarring by 50 years of age or younger. Brancato et al found a mean final BCVA of 20/640 in a series of 26 fellow eyes, before the era of antiangiogenic drugs. ${ }^{18}$ In a review by Clarkson and Altman, 13 patients had loss of two lines of vision after 6 months of follow-up, from which six eyes become legally blind due to bilateral disciform scars. ${ }^{12}$ In two separate reports, Singerman and Hatem and Arias et al reported similar observations with final BCVAs of 1.2 and $1.5 \log$ MAR $(\approx 20 / 300$ and $\approx 20 / 630)$ after a follow-up time ranging from 15 to 33 months. ${ }^{16,19}$ Factors that can directly affect final BCVA are the anatomical localization of the lesion, the patient's age at onset of symptoms, and concomitant eye diseases (PXE). 1,4,20

Since it is a relatively rare finding, most of the available information regarding its treatment come from short case series and single case reports. The pooling of individual data and further analysis and meta-analysis suggest that the most effective therapy is the use of intravitreal antiangiogenic drugs. These induce stabilization and visual improvement in $>80 \%$ of the cases. ${ }^{1}$ Previously used treatments such as photodynamic therapy and laser photocoagulation were successful in achieving visual stabilization. ${ }^{6,21,22}$ However, both have proven to be less effective than antiangiogenic therapy in achieving visual improvement and cannot prevent recurrences. ${ }^{21}$ Therefore, they are no longer considered standard of care, except under special circumstances.

After the first description by Teixeira et al, ${ }^{23}$ several reports have described the efficacy of bevacizumab, ranibizumab, and aflibercept in decreasing the leakage, macular thickness, and improving vision in patients with AS-CNV. 9,11,14,24 The pharmacological effect occurs rapidly. Evidence shows that the effect can be replicated several times despite recurrences in the short term (efficacy can be sustained up to 3 years after the first intravitreal dose). General consensus seems to be that AS-CNS behaves similarly to myopic CNS; it requires more injections per year that myopic CNS, but significantly fewer injections than that of CNV due to age-related macular degeneration. ${ }^{7}$

Under this circumstance, a PRN regimen seems like the natural choice, providing good control with less number of injections. However, in addition to the follow-up appointments and ancillary studies, the key for a successful PRN regimen relies on patient's awareness of recurrences along with early recognition of the symptoms and self-referral as soon as possible. Another shortcoming of a PRN regimen would be the lack of consensus regarding treatment decisions and follow-up criteria, even among physicians in the same practice. ${ }^{1}$

In the current case series, we are reporting the long-term results (53.8 months or 4.4 years) of a group of patients with AS-CNV who were treated with 1 loading dose of bevacizumab and a PRN regimen thereafter. The follow-up time was longer than in previous reports, which usually focus their conclusions on the 2- and 3-year mark.9,11 Despite the excellent initial visual recovery in 6 out of 7 study eyes, the final BCVA at the end of the time period surveyed was not different than the reported at baseline. Patients received on their study eye an average of six intravitreal injections during the follow-up (range 4-15), a number very similar to the reported by Alagöz et al (5.1, range 1-20), ${ }^{9}$ Tilleul et al (9.9, range $2-26),{ }^{11}$ Kang and Roh (5.7), ${ }^{25}$ and Finger et al (6.5). ${ }^{4}$ The mean time between injections (3.8 months) was similar as well (range $\approx 3-9$ months). ${ }^{9}$ And yet, our results were not as encouraging as in previous studies. The reasons of our poor outcome are not well understood. Nevertheless, there are several key factors in our series that might be responsible for why the improved visual acuity was not maintained. First, our patients were older at presentation than those included in other series (fifth decade of life vs fourth decade of life), ${ }^{1,9,14}$ and some waited more than a year before seeking treatment. It is possible that the lack of awareness and treatment delay could have led to a greater macular damage and jeopardized 
the functional outcome despite a positive initial response to treatment. Second, three of our patients were diagnosed with PXE histopathologically during the follow-up. There are evidences that suggest that $\mathrm{AS}-\mathrm{CNV}$ s related to PXE have higher recurrence and require more intravitreal doses of antiangiogenic before achieving stabilization. ${ }^{4}$ Therefore, in a small sample like ours, with a significant proportion of patients with PXE, the probability of a good functional outcome is lower, especially if considering that the number of intravitreal injections and the time between treatments were not different to previous reports of AS-CNV in nonPXE population.

One of the strengths of this case series is that we limited our survey to patients with at least 4 years of follow-up. This gave us a broader view of how PRN regimen affects visual outcome in patients with AS-CNV in the long term. Although there is no clear evidence for superiority of a fixed regimen versus a PRN regimen, our results suggest that in a disease in which recurrence is the hallmark, a PRN regimen might struggle in achieving a satisfactory functional outcome in the long term, especially in older population in which selfawareness of recurrence and prompt self-referral might be unintentionally delayed, decreasing the chance for visual stabilization. In contrast, there is no enough information regarding the long-term effects of a fixed regimen over a retinal condition in which atrophy and fibrosis are an important part of the natural history of the disease. ${ }^{4}$ Chronic blockade of vascular endothelial growth factor may induce a loss of its neuroprotective effect and accelerate chorioretinal atrophy. In addition, the risk of serious cardiovascular adverse effects might be higher, if there is concomitant PXE, due to a higher risk of atherosclerosis, mitral valve disease, and vascular abnormalities in the patients. ${ }^{2}$

Another strength of this retrospective case series is that it reflects more accurately the daily conditions under which these patients are treated. Despite all our efforts to protocolize treatment, the cases are not always developed in clinical studies for various reasons. Sometimes due to economic factors, patients get lost during follow-up. Patients who achieved visual stabilization after the first doses of antiangiogenic drug may minimize the probability of a recurrence and decide to skip appointments. The patient's geographical localization and the need for traveling long distance for accessing medical care may decrease compliance with follow-up and treatment. Therefore, the relevance of our results may have a deeper meaning.

Besides its limitation due to its retrospective nature and small sample, the study has other limitations that we would like to mention. The follow-up relied heavily on patient's self-referral. Despite having strict appointment schedules during the follow-up and precise instructions regarding how to recognize a recurrence and how and when to seek help, the time between recurrence onset and patient visit to the office is uncertain. Therefore, the probability that the visual outcome is a reflection of a lack of timely treatment rather than a failure of the PRN regimen is high. The inclusion of a patient of 71 years of age as part of this series may have biased the results, since the age factor can also have a role in CNV formation and macular deterioration. However, after reviewing the entire medical record, we did not find any report of age maculopathy or degeneration. At the end, we decided to include this case because, despite the uncommon age, the clinical presentation and evolution was typical AS-CNV.

In summary, AS are uncommon retinal findings, in which the concomitant development of CNV may severely impair vision. Although antiangiogenic treatment has proved highly effective in controlling the acute episodes, there is no clear evidence about which treatment regimen is best. Although anatomical and functional success has been reported in the short term with a PRN treatment regimen, its benefits seem to fade when the follow-up is delayed.

Currently, there is no enough evidence in order to support a fixed regimen and its long-term effect is unknown. A treat and extend regimen is a feasible third option, but more studies are needed before recommending its use.

\section{Disclosure}

The authors report no conflicts of interest in this work.

\section{References}

1. Gliem M, Finger RP, Fimmers R, Brinkmann CK, Holz FG, Charbel Issa P. Treatment of choroidal neovascularization due to angioid streaks: a comprehensive review. Retina. 2013;33(7):1300-1314.

2. Georgalas I, Tservakis I, Papaconstaninou D, Kardara M, Koutsandrea C, Ladas I. Pseudoxanthoma elasticum, ocular manifestations, complications and treatment. Clin Exp Optom. 2011;94(2):169-180.

3. Ozkaya A, Alkin Z, Faiz M, Yazici AT, Demirok A. Intravitreal bevacizumab for choroidal neovascularization secondary to angioid streaks: a report of two patients. Saudi J Ophthalmol. 2014;28(4):316-318.

4. Finger RP, Charbel Issa P, Schmitz-Valckenberg S, Holz FG, Scholl HN. Long-term effectiveness of intravitreal bevacizumab for choroidal neovascularization secondary to angioid streaks in pseudoxanthoma elasticum. Retina. 2011;31(7):1268-1278.

5. Al-Rashaed S, Arevalo JF. Long-term follow-up of choroidal neovascularization secondary to angioid streaks: case series and literature review. Clin Ophthalmol. 2012;6:1029-1034.

6. Pece A, Russo G, Ricci F, Isola V, Introini U, Querques G. Verteporfin photodynamic therapy combined with intravitreal triamcinolone for choroidal neovascularization due to angioid streaks. Clin Ophthalmol. 2010;4:525-530.

7. Gupta B, Elagouz M, Sivaprasad S. Intravitreal bevacizumab for choroidal neovascularisation secondary to causes other than age-related macular degeneration. Eye (Lond). 2010;24(2):203-213. 
8. Spaide RF. Peau d'orange and angioid streaks: manifestations of Bruch membrane pathology. Retina. 2015;35(3):392-397.

9. Alagöz C, Alagoz N, Ozkaya A, et al. Intravitreal bevacizumab in the treatment of choroidal neovascular membrane due to angioid streaks. Retina. 2015;35(10):2001-2010.

10. Vaz-Pereira S, Collaco L, De Salvo G, van Zeller P. Intravitreal aflibercept for choroidal neovascularisation in angioid streaks. Eye. 2016; 30(6):896.

11. Tilleul J, Mimoun G, Querques G, et al. Intravitreal ranibizumab for choroidal neovascularization in angioid streaks: four-year follow-up. Retina. 2016;36(3):483-491.

12. Clarkson JG, Altman RD. Angioid streaks. Surv Ophthalmol. 1982; 26(5):235-246.

13. Artunay O, Yuzbasioglu E, Rasier R, Sengul A, Senel A, Bahcecioglu H. Combination treatment with intravitreal injection of ranibizumab and reduced fluence photodynamic therapy for choroidal neovascularization secondary to angioid streaks: preliminary clinical results of 12-month follow-up. Retina. 2011;31(7):1279-1286.

14. Esen E, Sizmaz S, Demircan N. Intravitreal aflibercept for management of subfoveal choroidal neovascularization secondary to angioid streaks. Indian J Ophthalmol. 2015;63(7):616-618.

15. Lee JW, Lai JS, Yick DW, Tse RK. Retrospective case series on the long-term visual and intraocular pressure outcomes of phacomorphic glaucoma. Eye (Lond). 2010;24(11):1675-1680.

16. Singerman LJ, Hatem G. Laser treatment of choroidal neovascular membranes in angioid streaks. Retina. 1981;1(2):75-83.

17. Nakagawa S, Yamashiro K, Tsujikawa A, et al. The time course changes of choroidal neovascularization in angioid streaks. Retina. 2013;33(4):825-833.
18. Brancato R, Menchini U, Pece A, Davi G, Capoferri C. Laser treatment of macular subretinal neovascularizations in angioid streaks. Ophthalmologica. 1987;195(2):84-87.

19. Arias L, Pujol O, Rubio M, Caminal J. Long-term results of photodynamic therapy for the treatment of choroidal neovascularization secondary to angioid streaks. Graefes Arch Clin Exp Ophthalmol. 2006;244(6):753-757.

20. Savastano MC, Minnella AM, Zinzanella G, Falsini B, Caporossi A. Successful long-term management of choroidal neovascularization secondary to angioid streaks in a patient with pseudoxanthoma elasticum: a case report. J Med Case Rep. 2014;8:458.

21. Pece A, Avanza P, Galli L, Brancato R. Laser photocoagulation of choroidal neovascularization in angioid streaks. Retina. 1997;17(1): $12-16$.

22. Menchini U, Virgili G, Introini U, et al. Outcome of choroidal neovascularization in angioid streaks after photodynamic therapy. Retina. 2004;24(5):763-771.

23. Teixeira A, Moraes N, Farah ME, Bonomo PP. Choroidal neovascularization treated with intravitreal injection of bevacizumab (Avastin) in angioid streaks. Acta Ophthalmol Scand. 2006;84(6):835-836.

24. Tetikoglu M, Sagdik HM, Aktas S, Ozcura F. Intravitreal aflibercept for refractory choroidal neovascularization secondary to angioid streaks. Eye (Lond). 2016;30(6):894-895.

25. Kang S, Roh YJ. Intravitreal ranibizumab for choroidal neovascularisation secondary to angioid streaks. Eye (Lond). 2009;23(8): $1750-1751$.
Clinical Ophthalmology

\section{Publish your work in this journal}

Clinical Ophthalmology is an international, peer-reviewed journal covering all subspecialties within ophthalmology. Key topics include: Optometry; Visual science; Pharmacology and drug therapy in eye diseases; Basic Sciences; Primary and Secondary eye care; Patient Safety and Quality of Care Improvements. This journal is indexed on

\section{Dovepress}

PubMed Central and CAS, and is the official journal of The Society of Clinical Ophthalmology (SCO). The manuscript management system is completely online and includes a very quick and fair peer-review system, which is all easy to use. Visit http://www.dovepress.com/ testimonials.php to read real quotes from published authors. 